\title{
La prévision hydrométéorologique sur le bassin versant du Rhône en amont du Léman
}

\author{
Hydrometeorological forecast on the Rhone River Catchment upstream \\ of Lake Geneva \\ JAVIER GARCÍA HERNÁNDEZ \\ EPFL-ENAC-IIC-LCH, Station 18, CH-1015 Lausanne, Suisse \\ Tél : +41216932385; Fax : +41 2169322 64, e-mail : javier.garciahernandez@epfl.ch
}

JEAN-LOUIS BOILLAT

EPFL-ENAC-IIC-LCH, Station 18, CH-1015 Lausanne, Suisse

Tél : +41216932385; Fax : +412169322 64, e-mail : jean-louis.boillat@epfl.ch

FRÉDÉRIC JORDAN

e-dric.ch, Grand-Chemin 73, CH-1066 Epalinges

Tél : +41 217843313 ; Fax : +41 2178433 22, e-mail : fred.jordan@e-dric.ch

BENOÎT HINGRAY

CNRS, LTHE, BP 53, F-38 041 GRENOBLE Cedex 09

Tél : +33476825054 ; Fax : +334 768250 14, e-mail : benoit.hingray@hmg.inpg.fr

$L$

'objectif prioritaire du projet de $3^{e}$ Correction du Rhône est d'améliorer la protection de la vallée du Rhône contre les inondations à l'amont du lac Léman. Dans ce contexte, le projet MINERVE vise à optimiser la gestion des flux hydrauliques lors de fortes précipitations, en tirant profit du réseau d'aménagements hydroélectriques existant sur le bassin versant. Pour ce faire, un modèle de prévisions hydrométéorologiques a été développé, assorti d'un modèle d'aide à la décision pour l'exploitation préventive des aménagements.

\footnotetext{
$7^{\text {he main }}$ goal of the $3^{\text {rd }}$ Rhône Correction project is to improve the flood protection in the Upper Rhone River basin. In this context, the MINERVE project aims contributing to a better flow control during flood events, taking advantage from the multireservoirs system existing in the watershed. For this purpose, a hydrometeorological forecast model has been developed as well as a decision support tool for the hydropower plants preventive management.
}

\section{INTRODUCTION}

Le bassin versant du Rhône en amont du Léman possède une surface de $5520 \mathrm{~km}^{2}$ dont $700 \mathrm{~km}^{2}$ en couverture glaciaire (Figure 1). Il est caractérisé par la présence de nombreux aménagements hydroélectriques dont le débit équipé total est de $350 \mathrm{~m}^{3} / \mathrm{s}$. Les plus importants sont dotés de bassins d'accumulation saisonnière d'un volume total supérieur à 1195 Mio $\mathrm{m}^{3}$.

Sur ce bassin versant, les crues de 1987, 1993 et 2000 ont rappelé l'importance de la gestion du risque d'inondation lors d'événements exceptionnels, susceptibles d'occasionner des dommages importants consécutifs au débordement des cours d'eau et des bassins d'accumulation.

Ces événements ont aussi démontré que la capacité de rétention et de régulation des grands barrages est capable de laminer significativement les crues sur l'ensemble du réseau hydrographique. Considérant qu'une interprétation adéquate des prévisions météorologiques permet d'identifier les intempéries potentiellement dommageables, il devient possible d'organiser des interventions préventives appropriées.

Le projet MINERVE [1] poursuit le double objectif de gestion préventive des crues et de déclenchement d'alarme. Il s'appuie sur un outil numérique de simulation hydrologique, basée sur les prévisions météorologiques. Il est aussi doté d'un outil d'aide à la décision qui propose des scénarios de turbinage et de vidange préventifs dans le but d'optimiser l'effet de laminage des retenues. Finalement, les résultats des simulations prévisionnelles permettent de décider des déclenchements d'alertes, par l'intermédiaire de la cellule de crise du canton du Valais, et de coordonner les actions en cas de catastrophe annoncée. 


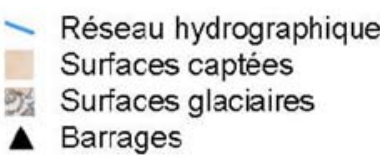

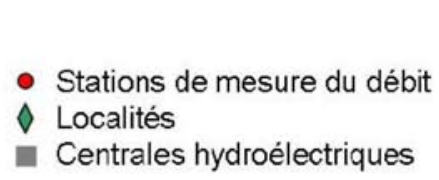

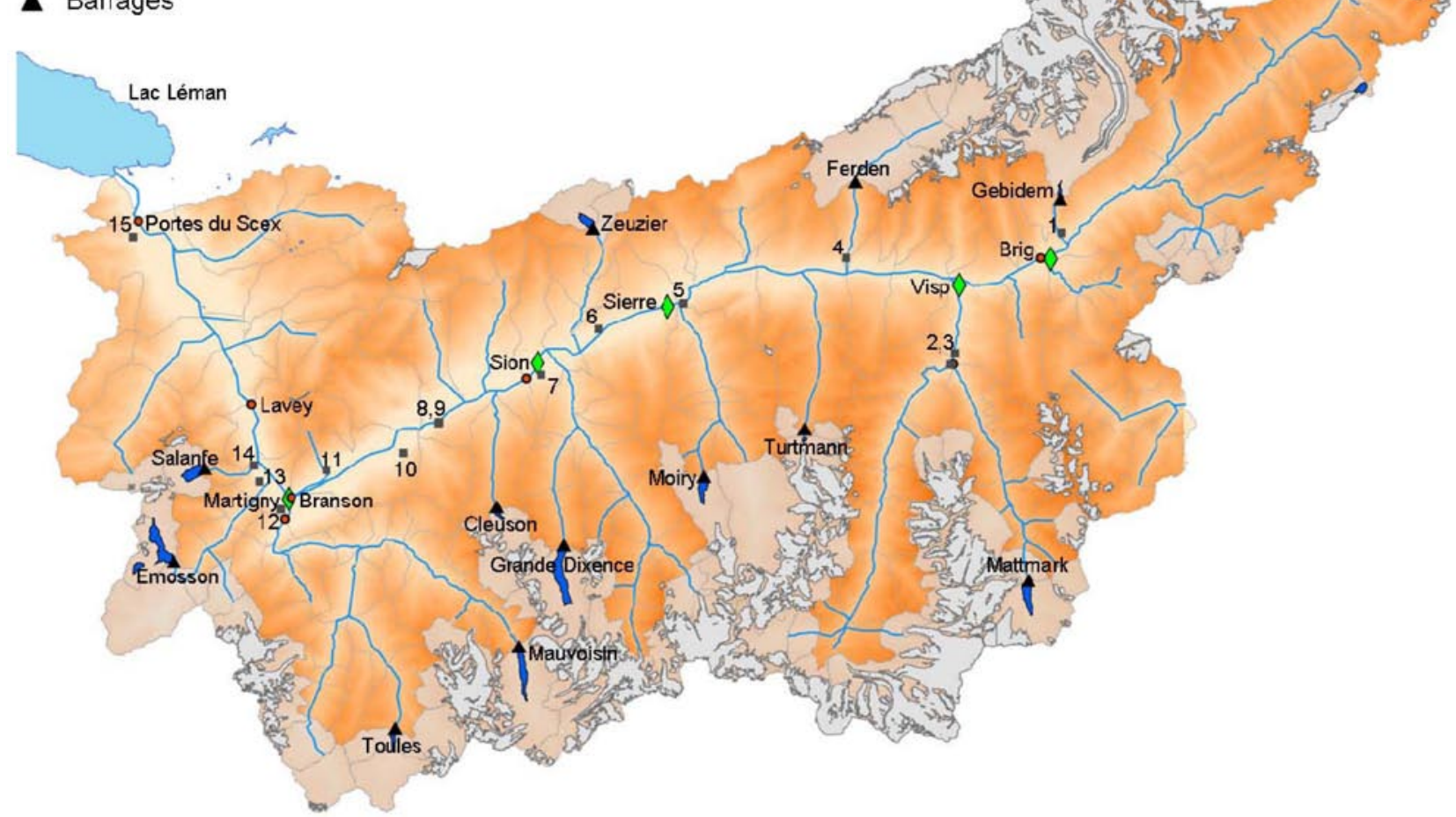

Figure 1 : Bassin versant du Rhône à l'amont du Lac Léman avec retenues et centrales : 1) Bitsch, 2) Ackersand, 3) Stalden, 4) Steg, 5) Chippis (Navisence), 6) St. Léonard, 7) Chandoline, 8) Nendaz, 9) Bieudron, 10) Riddes, 11) Fully, 12) La Batiaz, 13) Vernayaz, 14) Miéville, 15) Vouvry

\section{II — LE MODÈLE HYDROLOGIQUE}

Le bassin versant du Rhône a été discrétisé en 239 sousbassins versants d'une surface moyenne de $23 \mathrm{~km}^{2}$, de manière à tenir compte des nombreux ouvrages de captage situés en altitude. Le concept du modèle hydrologique utilisé pour estimer les débits à l'exutoire de chaque sous bassin [2] se base sur les modèles Socont [3] et GSM-Socont [4 \& 5] développés par le Laboratoire Hydrologie et Aménagements (Hydram) de l'EPFL. La propagation dans le réseau hydrographique des débits produits par les différents sousbassins est effectuée avec un modèle de propagation d'onde cinématique.

Pour la partie hydrologique de la modélisation, chaque sous-bassin versant est divisé en deux régions, une glaciaire et l'autre non glaciaire, toutes deux découpées en bandes d'altitude. Pour chaque bande d'altitude, un modèle de neige, composé d'un double réservoir (neige et eau contenue dans la couche de neige), permet de suivre l'évolution temporelle de la hauteur et du degré de saturation de la neige. Les précipitations et températures horaires sont estimées à cet effet pour chaque bande d'altitude. La fonte de neige, calculée selon une formule de type degré-jour, produit une pluie équivalente à partir d'un seuil de saturation.
Dans le cas d'une bande non glaciaire, cette pluie équivalente alimente le modèle d'infiltration et de transfert. Ce modèle est composé de deux réservoirs non-linéaires en parallèle, produisant respectivement les composantes lentes et rapides de l'écoulement à l'exutoire de la bande. Dans le cas d'une bande glaciaire, la pluie équivalente résultant de la fonte éventuelle de neige est transférée à l'exutoire par un réservoir linéaire. Lorsqu'il n'y a plus de neige sur une bande glaciaire, un modèle de fonte de glace de type degréjour produit un débit également transféré à l'exutoire par un réservoir linéaire. Le débit total d'un sous-bassin versant est la somme des diverses contributions de chaque bande d'altitude.

\section{LE SYSTÈME DÉTERMINISTE DE PRÉVISION ET DE GESTION DES CRUES}

Le modèle MINERVE exploite actuellement les prévisions météorologiques déterministes provenant du modèle COSMO-7 (Consortium for Small-scale Modeling) de l'Office Fédéral de Météorologie et Climatologie (MétéoSuisse). La prévision COSMO-7 est établie à partir du modèle glo- 
bal ECMWF (European Centre for Medium-Range Weather Forecasts) et couvre la majeure partie de l'Europe occidentale et centrale. La prévision est réalisée deux fois par jour à un horizon de 72 heures, selon une grille d'environ $6.6 \mathrm{~km}$ avec 60 niveaux verticaux.

Pour transformer les prévisions de pluie et température en débit, le modèle hydrologique semi-distribué décrit ci-dessus a été utilisé. Ce modèle est construit à l'aide de l'outil de simulation hydrologique et hydraulique Routing System [6]. Développé spécialement pour ce projet, ce logiciel a été conçu pour simuler la formation et la propagation des écoulements dans des systèmes à surface libre. Il autorise la modélisation hydrologique et hydraulique par une approche orientée objets, selon un schéma de type conceptuel semidistribué (cf. Figure 2). Il permet la prise en compte de processus hydrologiques particuliers tels que la fonte de neige et glaciaire, les écoulements de surface et de sub-surface, le routage dans les retenues, les galeries et les cours d'eau et aussi la modélisation d'ouvrages régulés tels que vannes, évacuateurs réglables, prises d'eau, turbines ou pompes.

Le modèle MINERVE est capable d'établir la prévision de crues en temps réel, couplant les informations météorologiques avec le modèle hydrologique et de transfert. Le suivi en temps réel des écoulements peut également être fait sur la base de mesures antécédentes de précipitations et de température.

Chaque fois qu'une nouvelle prévision météorologique est fournie, la prévision hydrologique est rafraîchie, en occultant les résultats de la précédente. Cette procédure est illustrée à la Figure 3, où l'observation à l'exutoire, la simulation avec les données météorologiques observées et le résultat des prévisions météorologiques successives sont présentés.

De la même façon que la prévision météorologique est régulièrement rafraichie, la prévision hydrologique peut aussi être ajustée au début de chaque nouvelle simulation par une mise à jour des variables d'état, pour réduire les erreurs de prévision liées au modèle hydrologique [2].

Finalement, un modèle d'aide à la décision propose des stratégies d'intervention pour la gestion préventive des aménagements hydroélectriques dans le but d'éviter les inondations sur le bassin versant. Ce modèle s'appuie sur des objectifs pré-établis et tient compte des restrictions d'exploitation. Ce système expert [7] poursuit un double but. Le premier vise à libérer préventivement un volume de stockage dans les retenues et à interrompre les opérations de turbinage et/ou de vidange durant la pointe. Le deuxième consiste à

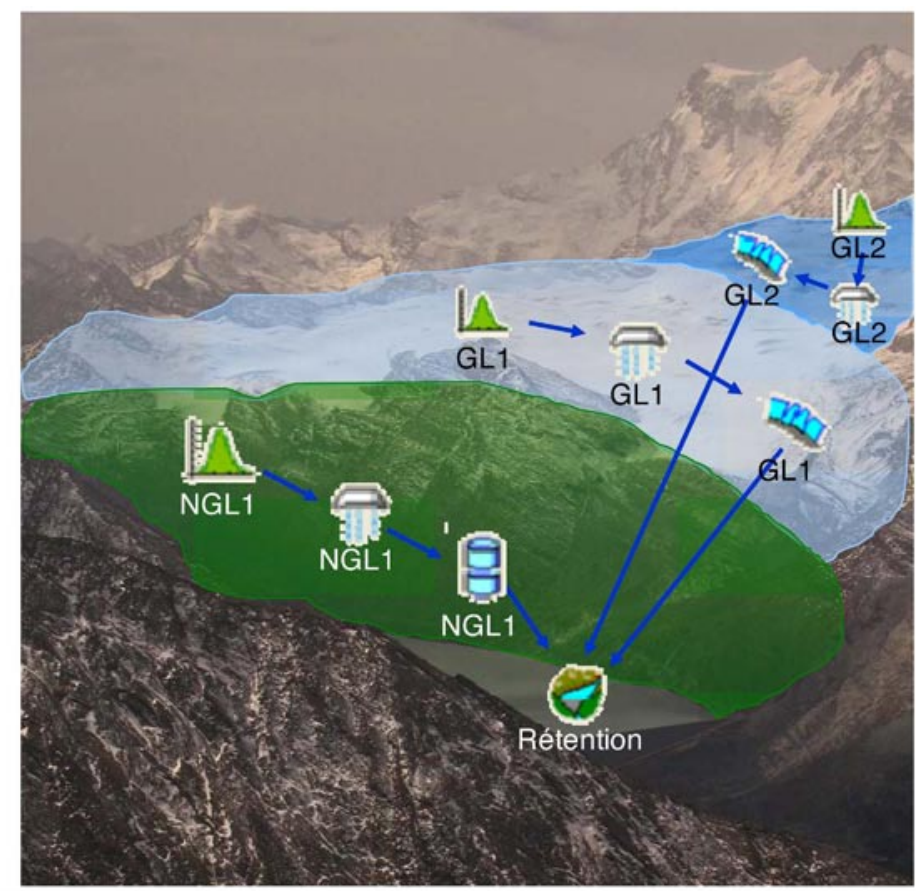

\begin{tabular}{|c|c|c|c|}
\hline L & Station météo virtuelle & 붐 & Ecoulements de surface et subsurface \\
\hline & Rétention & & Gestion de la fonte de glace \\
\hline 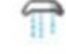 & Gestion du stock de neige & GL & Bande d'altitude glaciaire \\
\hline$\rightarrow$ & Transfert des flux & NGL & Bande d'altitude non glaciaire \\
\hline
\end{tabular}

Figure 2 : Modélisation hydrologique fonctionnelle selon le concept Routing System à l'exemple du sous-bassin versant de Mattmark, avec découpage en bandes d'altitude des zones glaciaires et neigeuses 


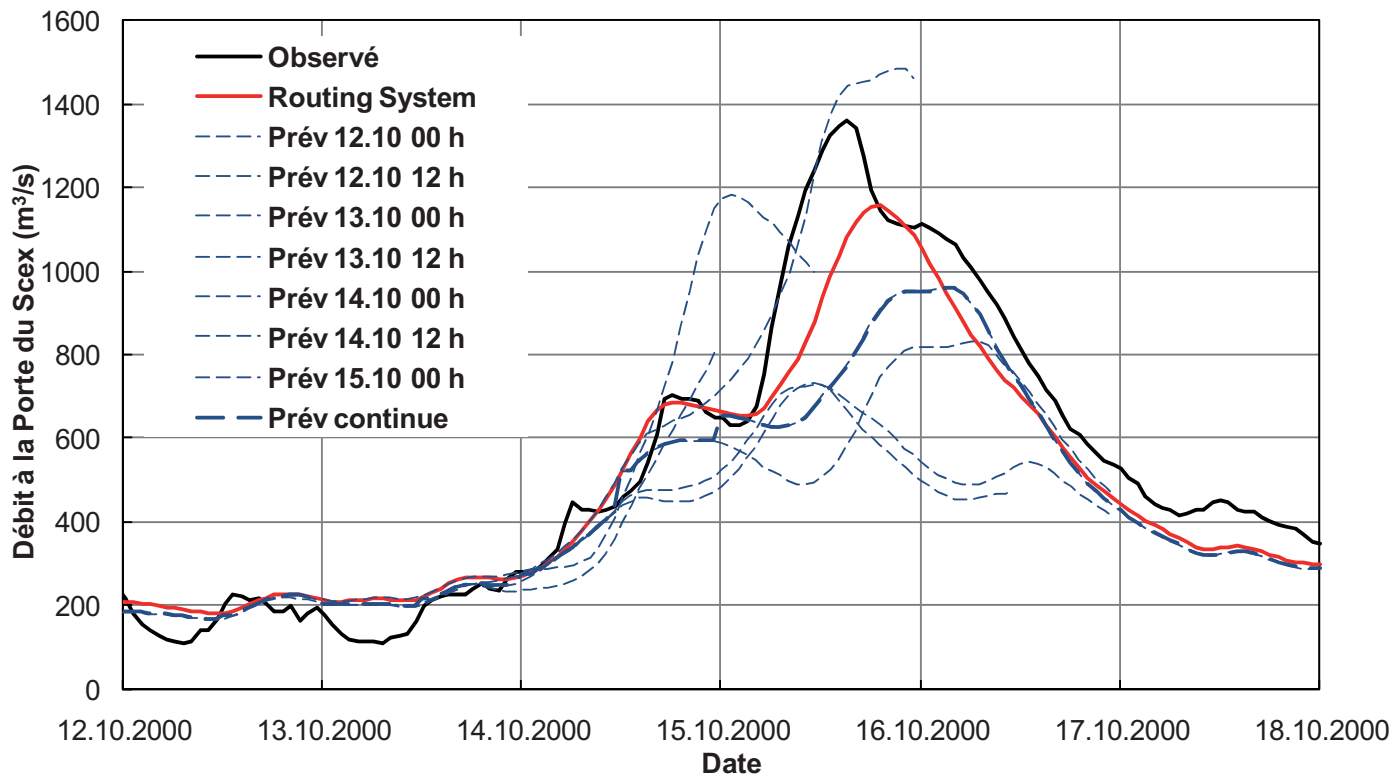

Figure 3 : Simulations successives des prévisions météorologiques (Prév), prévision continue en conservant les 12 premières heures de chaque simulation (Prév continue), hydrogramme avec les données météorologiques observées (Routing System) et débit observé (Observé) à la Porte du Scex

optimiser la reconstitution des stocks à la fin de la crue, de façon à éviter les pertes d'eau.

Les débits prévus en certains points de contrôle du réseau hydrologique sont utilisés comme entrée du modèle d'optimisation. Ce dernier exploite également les niveaux des retenues ainsi que l'état de fonctionnement des différents ouvrages (turbines, pompes, prises d'eau, vidanges de fond). Grâce à ces données, le modèle d'optimisation calcule le programme des opérations de vidange par turbinage ou par ouverture des organes de vidange, de manière à contrôler au mieux le débit des cours d'eau aval. A la fin de cette procédure, les opérations de vidange préventive proposées par le modèle sont applicables jusqu'à la prochaine prévision météorologique (12 heures plus tard) ou jusqu'à la prochaine mise à jour du modèle. Un exemple de résultat obtenu par cette démarche est illustré sur la Figure 4.

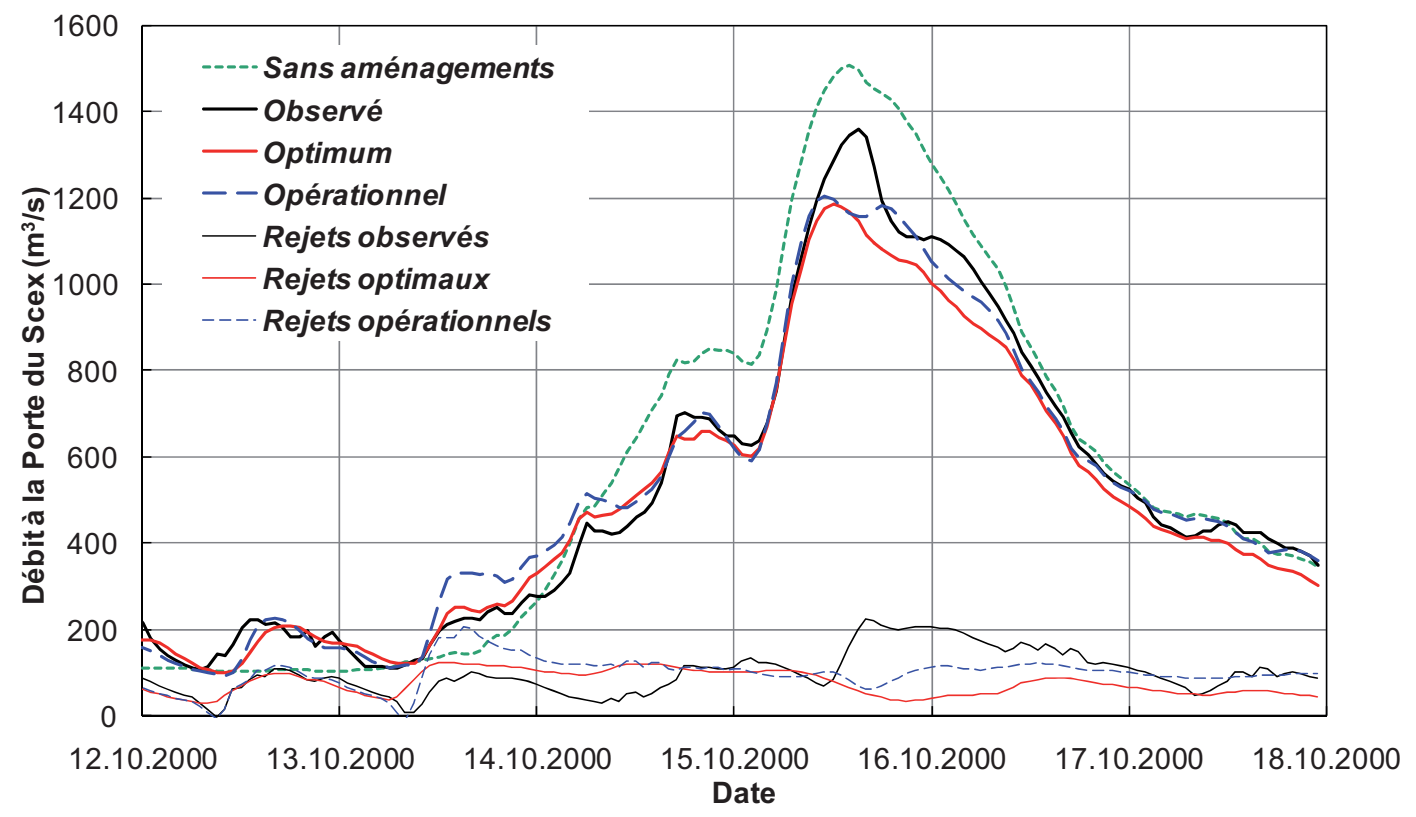

Figure 4 : Hydrogrammes du Rhône à l'arrivée dans le Léman lors de la crue d'octobre 2000. Simulations sans retenues (Sans aménagements), avec une gestion basée sur les données météorologiques observées (Optimum) et avec une gestion basée sur les prévisions météorologiques (Opérationnel), en comparaison de la crue observée (Observé) 
L'utilisation des aménagements hydroélectriques pour le contrôle des crues est une opération risquée. Si les objectifs du décideur sont prioritairement de limiter les débits dans les cours d'eau à une valeur acceptable, ils doivent aussi considérer l'économie des moyens. Il s'agit ainsi de limiter les pertes économiques liées aux opérations préventives lors des crues. Il est connu que les prévisions hydro-météorologiques quantitatives sont entachées d'incertitudes importantes, en particulier pour ce qui concerne les précipitations. La caractérisation des incertitudes peut être partiellement effectuée par l'utilisation de prévisions d'ensemble - cf. section IV. Dans tous les cas, il est recommandé de ne pas utiliser ces données sans contrôle. Heureusement, l'utilisation simultanée d'un outil de prévision hydrologique et d'un système d'informations en temps-réel offre une complémentarité qui permet au décideur d'éviter la dérive.

Le processus général lié à la prise de décision en situation de crue suit un déroulement logique [8]. Lorsqu'une « alarme-eau » est émise par MétéoSuisse, une première prévision hydrologique est effectuée, basée sur la prévision météorologique la plus récente et sur l'état initial du bassin versant. Un diagnostic de la situation hydrologique du bassin versant est alors posé, mettant en évidence les points potentiellement critiques sur le réseau hydrographique. A cet instant, il est important de bien identifier quelles conditions hydrométéorologiques mènent à d'éventuels problèmes dans le bassin versant. Il convient en particulier de souligner les valeurs moyennes des précipitations et températures prévues qui conduisent à des débits prévisionnels élevés dans certains cours d'eau du bassin versant.

Tant que la situation n'est pas critique, il convient d'attendre jusqu'à la prochaine prévision météorologique ou jusqu'à la prochaine mise à jour du modèle hydrologique. Pendant cette période, les débits, mais aussi et surtout les précipitations et températures prévues et observées sont comparés et les différences entre valeurs prévues et observées sont consignées. Sur la base de ces informations, du temps de réponse des bassins et sous-bassins versants ainsi que des durées nécessaires pour les opérations préventives, il est possible d'établir les règles suivantes pour que l'application des opérations de vidange préventive produise les effets escomptés : - en cas de surestimation (des précipitations par exemple), il est possible d'attendre avant de procéder à des opérations de vidange préventive, éventuellement jusqu'à la prochaine prévision ou au moins jusqu'à l'apparition de précipitations plus fortes, toujours en considérant le temps de réponse du bassin versant ;

- en cas de sous-estimation des débits, précipitations ou températures, la situation devient critique plus tôt que prévu. Une première cause de cet état de fait peut être le décalage des prévisions météorologiques. Une autre raison peut résulter de la sous-estimation des précipitations et/ou des températures. Dans ce cas, les observations radar peuvent contribuer à déterminer s'il s'agit d'un décalage temporel ou d'une sous-estimation des valeurs météorologiques. Il est alors nécessaire de préparer les procédures d'urgence (opérations de vidange préventive, alarmes, information, évacuations, etc...) pour la réduction des dommages dans le bassin versant, et de procéder au plus vite à une nouvelle prévision de débit.
Dès qu'une nouvelle information est disponible (nouvelle observation, nouvelle prévision), la procédure de décision doit à nouveau être appliquée, et ceci jusqu'à ce que l'alerte soit terminée.

Cette procédure met en évidence deux modes différents de fonctionnement et d'utilisation du modèle. Le premier correspond à une exploitation normale, quasi en continu, qui permet de suivre l'évolution de la situation hydrologique sur le bassin versant. Le deuxième répond à une situation de crise où le but est d'informer et de faciliter les prises de décisions pour la protection des personnes et des biens contre les crues.

A ce stade du projet, les cantons du Valais et de Vaud disposent d'un modèle numérique capable de simuler les écoulements dans le réseau hydrographique naturel et aménagé, ainsi que de proposer des consignes d'exploitation préventives destinées à la protection contre les crues. En septembre 2006, le système MINERVE a été exploité pour la première fois en situation d'alarme-eau. Après une alerte de MétéoSuisse, plusieurs prévisions hydrologiques à 72 heures ont été réalisées sur le bassin versant pour établir un bilan de la situation sur le Rhône et les aménagements hydroélectriques, qui a révélé une surestimation des prévisions. La procédure de suivi décrite plus haut a néanmoins permis d'éviter la prise de décisions erronées.

\section{PRISE EN COMPTE DES PRÉVISIONS D'ENSEMBLE}

Une prévision déterministe est susceptible de bercer l'utilisateur d'une illusion de certitude, qui peut facilement aboutir à une action sous-optimale [9]. Quand bien même de nombreuses améliorations ont été faites dans la prévision déterministe, particulièrement à court terme, la performance n'est pas toujours suffisante pour une application à la gestion du risque lors de fortes précipitations. Des améliorations substantielles doivent encore être effectuées pour améliorer la fiabilité des prévisions à moyen terme et renforcer la prise de décisions.

Les prévisions d'ensemble (Ensemble Prediction System, EPS) apportent un éclairage nouveau à cette question, avec certains avantages potentiels. Elles sont un complément utile aux prévisions déterministes en ce sens qu'elles fournissent une évaluation de l'incertitude associée aux événements considérés [10]. Elles peuvent par suite être employées pour l'évaluation de différents critères concernant la gestion des aménagements ou les alertes d'inondation, en rendant possible la prise en compte d'un risque explicite. L'EPS constitue l'un des domaines les plus prometteurs de la recherche météorologique pour compenser l'incertitude liée aux prévisions déterministes [11].

Depuis les années 90, le modèle de circulation du Centre européen pour les prévisions météorologiques à moyen terme (European Center for Medium range Weather Forecasting, ECMWF) produit des prévisions météorologiques d'ensemble. Elles résultent de différents scénarios sur l'état initial de l'atmosphère, principale source d'incertitude pour les modèles. Elles se composent actuellement de 50 prévisions 
perturbées et d'une prévision non-perturbée à un horizon de quinze jours avec un pas de temps de $720 \mathrm{~s}$. Les prévisions d'ensemble sont produites deux fois par jour à 00 et 12 UTC. Pour la résolution horizontale dans l'atmosphère libre, une méthode spectrale est employée. Elle correspond à une longueur de grille d'environ 25 kilomètres. Sur la verticale, l'atmosphère est divisée en 91 couches d'altitude suivant le relief et jusqu' à $0.01 \mathrm{hPa}$ (environ 80 kilomètres).

MétéoSuisse, partenaire du projet MINERVE, produit les prévisions météorologiques d'ensemble COSMO-LEPS. Ce modèle EPS a été développée par le consortium de COSMO (Consortium for Small-scale Modeling) depuis 2002. Le système permet de combiner les avantages de l'approche ensembliste avec la haute résolution des intégrations du modèle [12]. Le système COSMO-LEPS est en conséquence utile pour la prévision d'événements de précipitations extrêmes dans une perspective ensembliste. Il fournit des prévisions d'ensemble journalières à très haute résolution (taille de maille horizontale de 10 kilomètres et 40 couches verticales) sur un ensemble de 16 scénarios pour l'Europe centrale et méridionale à un horizon de 132 heures. Ces 16 scénarios sont une sélection représentative des 102 prévisions d'ensemble du modèle global ECMWF (51 membres à $00 \mathrm{~h}$ et 51 à $12 \mathrm{~h}$ UTC), lesquelles sont utilisées pour l'initialisation et le calcul des conditions de bords de COSMO-LEPS.

Le but de COSMO-LEPS est donc l'amélioration des prévisions d'événements extrêmes localisés à court et moyen terme, en particulier quand les processus liées à l'orographie et au contexte atmosphérique régional jouent un rôle crucial [13]. Comme ses perturbations dérivent de celles de l'ECMWF, le système est particulièrement indiqué pour la gamme du court et moyen terme (jours 3 - 5).

Pour les prévisions à plus court terme, les prévisions déterministes COSMO-7 et COSMO-2 de MétéoSuisse sont un complément intéressant de COSMO-LEPS. Le modèle local COSMO-2, alimenté initialement par COSMO-7, couvre la région alpine avec la Suisse au milieu et est calculé sur un espacement de grille d'environ 2.2 kilomètres. Il est calculé huit fois par jour pour une durée de 24 heures. Ces deux modèles permettent l'utilisation des dernières conditions existantes pour la prévision à court terme. Les caractéristiques de ces différents modèles de prévision météorologiques sont résumées dans le Tableau 1.

Comme alternative aux prévisions météorologiques fournies par MétéoSuisse et pour fournir une information additionnelle aux décideurs, un modèle d'adaptation des prévisions météorologiques basé sur une méthode de type analogues pour l'identification de scénarios de précipitations est en cours de développement, selon une approche similaire à celle développée par Obled et al. [14]. Ce modèle de descente d'échelle statistique utilisera les champs, à l'échelle synoptique, de diverses variables atmosphériques mieux simulées par les modèles météorologiques que les variables de surface telles que les précipitations.

L'utilisation des prévisions météorologiques d'ensemble COSMO-LEPS permet de produire des prévisions hydrologiques d'ensemble. De telles applications ont déjà été faites et évaluées en Europe. A titre d'exemple, De Roo et al. [15] ont simulé des crues avec un modèle hydrologique et des prévisions d'ensemble (EPS) pour l'Europe (European Flood Forecast System, EFFS) et obtenu des résultats encourageants. Roulin [16] a développé un système hydrologique de prévisions d'ensemble basé sur l'ECMWF, examinant les résultats sur deux sous-bassins belges. L'étude a comparé les résultats obtenus aux valeurs déterministes et conclu que les prévisions hydrologiques d'ensemble ont une meilleure performance. Finalement, Olsson [17] a aussi fait une évaluation et un calage des prévisions hydrologiques d'ensemble en Suède en utilisant ECMWF et un modèle HBV.

Dans le système MINERVE, les données hydro-météorologiques observées et les prévisions météorologiques d'ensemble sont téléchargées en temps réel. Le modèle est capable de faire des simulations multiples, avec une procédure de mise à jour du modèle hydrologique en temps réel, tenant compte des mesures disponibles aux différents points du bassin versant.

Des hydrogrammes provenant d'une prévision EPS sont présentés à la Figure 5, ainsi que le débit moyen pondéré et le débit observé. Une analyse détaillée de ces nouvelles prévisions hydrologiques d'ensemble sera réalisée dans le but d'évaluer l'incertitude associée aux hydrogrammes simulés. Un ensemble représentatif de scénarios hydrologiques, y compris le minimum et le maximum, sera utilisé pour le modèle d'aide à la décision.

\section{$V \square$ GESTION DU RISQUE}

Les méthodes d'analyse de risque et de fiabilité ont gagné en importance au cours des dernières années dans les décisions relatives à l'ingénierie civile [18]. Elles permettent aux décideurs de connaître la rationalité de leurs décisions, ce qui est particulièrement important dans les prévisions de crues, où les prévisions hydro-météorologiques sont particulièrement incertaines. L'évaluation du risque est ainsi considérée comme un aspect nécessaire dans ce type de prévisions [19].

Tableau 1 : Caractéristiques des différents modèles COSMO de MétéoSuisse

\begin{tabular}{|l|c|c|c|}
\hline & COSMO-LEPS & COSMO-7 & COSMO-2 \\
\hline Type de prévision & Probabiliste $(16$ membres $)$ & Déterministe & $2.2 \cdot 2.2 \mathrm{~km}$ \\
\hline Résolution & $10 \cdot 10 \mathrm{~km}$ & $6.6 \cdot 6.6 \mathrm{~km}$ & 60 \\
\hline Niveaux verticaux & 40 & 60 & $24 \mathrm{~h}$ \\
\hline Horizon & $132 \mathrm{~h}$ & $72 \mathrm{~h}$ & $3 \mathrm{~h}$ \\
\hline Actualisation & $24 \mathrm{~h}$ & $12 \mathrm{~h}$ & \\
\hline
\end{tabular}




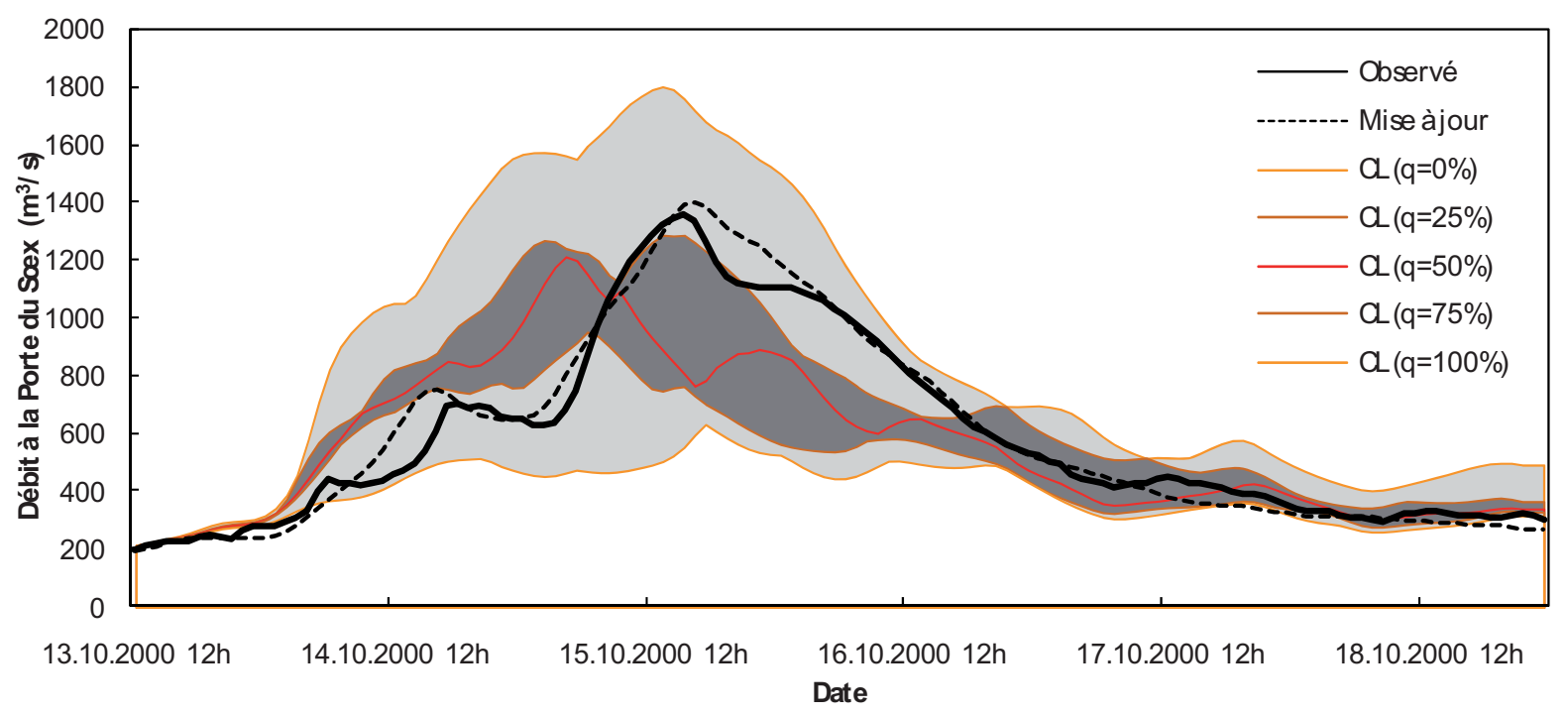

Figure 5 : Prévisions hydrologiques d'ensemble sur le Rhône à la Porte du Scex pour la prévision COSMO-LEPS du 13.10.2000 $12 \mathrm{~h}(\mathrm{CL})$, simulation avec les données météorologiques observées et la mise à jour des conditions initiales (Mise à jour), débit observé (Observé)

La Commission européenne a proposé en 2006 une directive concernant l'évaluation et la gestion des inondations [20] où l'évaluation préliminaire du risque d'inondation, les cartes de risque d'inondation et les plans de gestion de ce risque sont considérés comme nécessaires et fondamentaux.

Les systèmes d'aide à la décision (Decision Support System, DSS) sont des outils conçus pour la prise de décisions fondées sur différentes alternatives dépendant de valeurs estimées. Ils sont devenus incontournables pour l'optimisation des réseaux complexes de réservoirs à objectifs multiples tels que la production d'électricité, l'approvisionnement en eau ou le contrôle des crues. Ce dernier point est toujours associé à un degré d'incertitude lié à la météorologie, l'hydrologie ou même à une insuffisance de données, devant être convenablement évalué et traité dans le développement d'un outil d'aide à la décision efficace. Des nombreux travaux ont contribué à la prise de décisions multi-objectifs sous incertitude, mais une méthodologie appropriée pour la gestion des crues en temps réel doit encore être développée [21].

Dans l'actuel projet, le système d'aide à la décision devra identifier les stratégies optimales de gestion des aménagements hydroélectriques en tenant compte de scénarios probabilistes et fournir des diagnostics et des méthodes pour la gestion des crues. Ce nouveau DSS, baptisé MINDS (Minerve Interactive Decision Support) est en cours d'élaboration. Il constitue un développement scientifique essentiel du projet, par l'intégration systématique du concept de risque.

Un schéma opérationnel de ce nouveau développement du système MINERVE avec l'intégration de MINDS est présenté à la Figure 6. Après « l'alerte-eau » de MétéoSuisse, une analyse des prévisions météorologiques et hydrologiques, ainsi que de la situation actuelle des aménagements hydroélectriques (A.H.) est effectuée. Une première évaluation du danger est réalisée dans le but d'identifier si le système présente des problèmes de débordement aux points de contrôle du réseau et si une gestion des aménagements est nécessaire pour la réduction des risques d'inondation. Dans le cas requis, MINDS proposera des solutions optimales en prenant toujours en compte le risque, les fonctions des coûts associés ainsi que les incertitudes liées aux prévisions hydro-météorologiques. Dès qu'une nouvelle prévision ou de nouvelles données sont fournies, une nouvelle analyse de la situation est effectuée et une nouvelle gestion proposée.

Les principales entrées de MINDS sont les résultats des scénarios hydrologiques d'ensemble (hydrogrammes, niveaux des réservoirs,...) ainsi que les consignes de fonctionnement des aménagements hydroélectriques (capacité de turbinage et de vidange, règles d'opérations préventives,...). Une autre entrée importante pour l'outil MINDS est le coût associé aux inondations et/ou aux opérations préventives des aménagements hydroélectriques. Le coût des dommages dus aux inondations potentielles est évalué de même que celui résultant d'une gestion inappropriée des aménagements hydroélectriques. Cette évaluation de coûts repose sur une analyse approfondie des capacités hydrauliques de réseau naturel.

En conclusion, l'outil MINDS fournira l'optimisation en temps réel du système. Les prestations de MINDS pour la gestion des inondations peuvent être récapitulées comme suit :

- Mise à jour et visualisation automatiques de données

- Optimisation en temps réel pour une prise de décision en temps réel

- Visualisation des règles opérationnelles et des hydrogrammes résultants

- Évaluation des performances des scénarios d'opérations et du risque associé

- Possibilité de modification de certaines règles par le décideur

- Possibilité de tenir compte d'une information nouvelle 


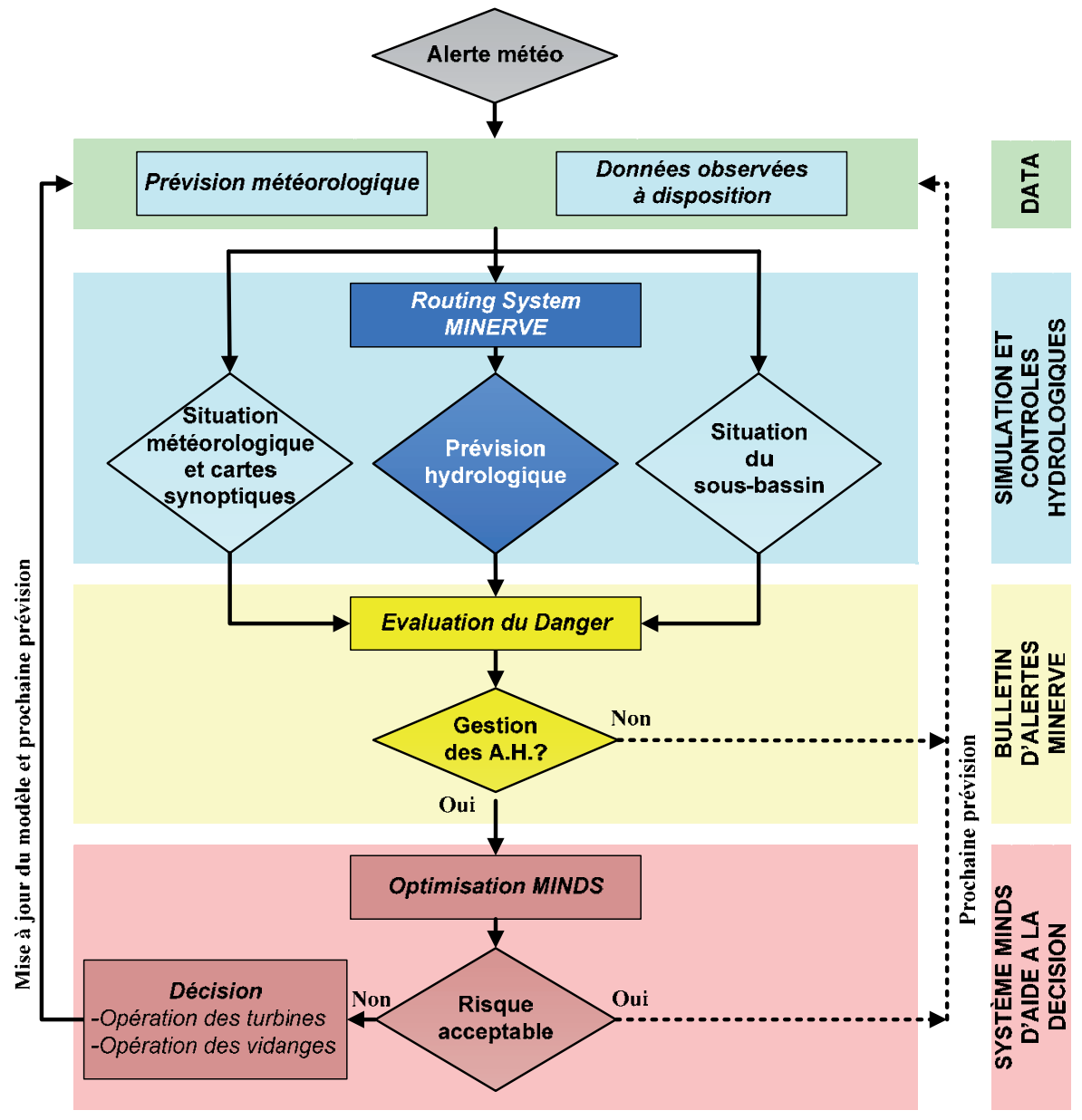

Figure 6 : Schéma opérationnel de MINERVE

Pour être utilisables efficacement, les résultats de MINDS doivent être accessibles de manière simple et compréhensible, avec un support graphique adéquat. La Figure 7 présente une maquette du tableau de bord contenant l'information principale nécessaire aux décideurs en situation de crue : hydrogrammes aux points de contrôle, avec et sans interventions préventives pour différents scénarios ; évaluation du risque associé aux scénarios et décisions proposés ; carte synoptique du bassin versant et de ses affluents, état des aménagements hydroélectriques, distribution spatiale de la précipitation et de la température et surtout les règles opérationnelles optimales données pour chaque aménagement hydroélectrique.

\section{CONCLUSIONS ET PERSPECTIVES}

Le modèle MINERVE développé pour les Cantons du Valais et de Vaud est opérationnel depuis 2006. Il permet de simuler les flux hydrauliques dans le réseau hydrographique du Rhône alpin et de proposer des consignes d'exploitation préventive pour la protection contre des inondations. Le système contribue à la prise de décisions prioritaires concernant la gestion des réservoirs des grands barrages pour renforcer la sécurité du territoire. Il permet également d'établir un tableau synoptique de la situation hydro-météorologique sur l'ensemble du bassin versant.

Le système actuel produit des résultats satisfaisants. Néanmoins, des améliorations substantielles sont en cours de développement pour augmenter sa fiabilité et faciliter les décisions pendant les événements de crue. La poursuite du projet permettra d'intégrer l'information liée aux derniers progrès technologiques en exploitant les prévisions d'ensemble de COSMO-LEPS (Consortium for Small-scale Modeling - Limited-area Ensemble Prediction System). Le but recherché est l'obtention d'un système plus robuste et plus fiable, intégrant l'incertitude de la prévision hydro-météorologique dans le processus de décision.

Le projet MINERVE est développé en partenariat par l'Office Fédéral de l'Environnement, les Services des Routes et Cours d'Eau, de l'Energie et des Forces Hydrauliques du Canton du Valais et par le Service des Eaux, Sols et Assainissement du Canton de Vaud. MétéoSuisse fournit les prévisions météorologiques et les sociétés hydroélectriques communiquent les informations relatives à leurs aménagements. Les bureaux d'ingénieurs HydroCosmos et Epicard contribuent aux aspects informatiques. Les développements scientifiques sont confiés à deux entités de l'Ecole Poly- 


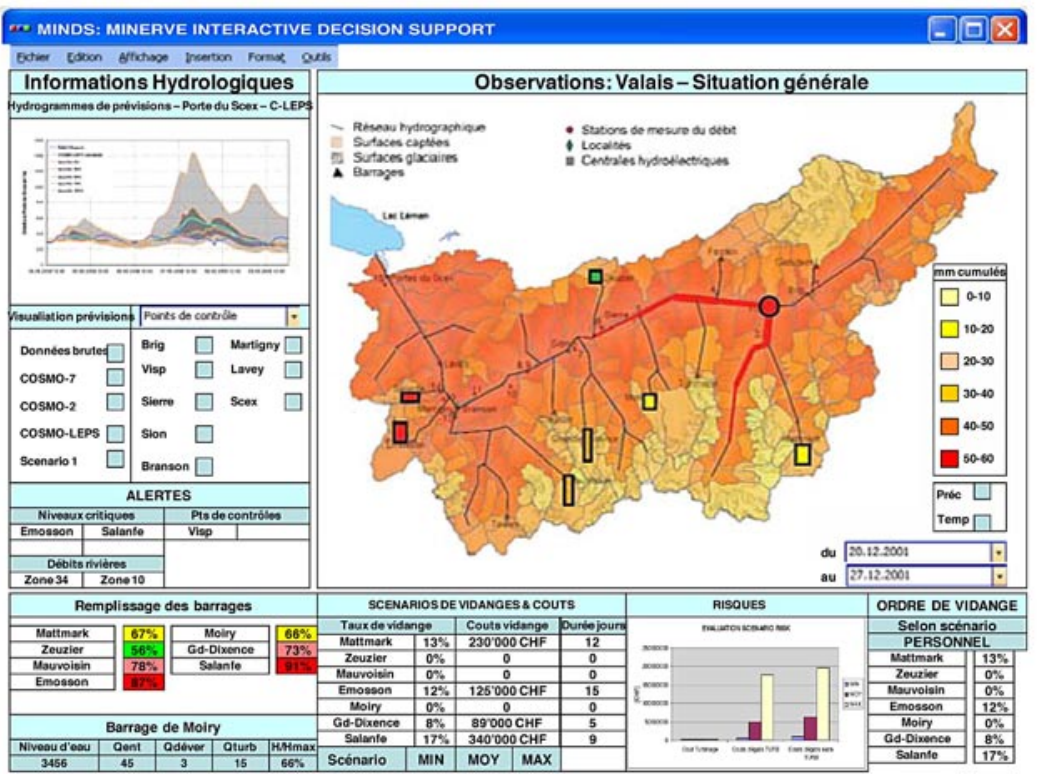

Figure 7 : Maquette de tableau de bord de MINDS (MINERVE Interactive Decision Support)

technique Fédérale de Lausanne (EPFL), le Laboratoire de Constructions Hydrauliques et le Laboratoire d'Ecohydrologie, ainsi qu'à l'Institut de Géomatique et d'Analyse du Risque de l'Université de Lausanne (UNIL).

\section{VII — RÉFÉRENCES}

[1] Boillat J.-L. (2005) - L'influence des retenues valaisannes sur les crues Le projet MINERVE Communication LCH No21. Conférence sur la recherche appliquée en relation avec la $3 e$ Correction du Rhône - Nouveaux développements dans la gestion des crues, Martigny. 87-101

[2] Hamdi Y., Hingray B., Musy A. (2005) - Un modèle de prévision hydro-météorologique pour les crues du Rhône supérieur en Suisse. Wasser, Energie and Luft. 11-12 pp. 325-332

[3] Bérod D. (1994) - Contribution à l'estimation des crues rares à l'aide de méthodes déterministes. Apport de la description géomorphologique pour la simulation des processus d'écoulement. PhD Thesis $N^{\circ} 1319$, Ecole Polytechnique Fédérale de Lausanne.

[4] Schäfli B., Hingray B., Niggli M., Musy A. (2005) — A conceptual glacio-hydrological model for high mountainous catchments. Hydrology and Earth System Sciences Discussions. 2 73-117

[5] Hingray B., Mezghani A., Schaefli B., Niggli M., Faivre G., Guex F., Hamdi Y., Musy A. (2006) - Estimation des débits de crue du Rhône à Porte du Scex et autres points amont caractéristiques. Rapport final du projet CONSECRU 2. Laboratoire Hydrologie et Aménagements. EPFL, Lausanne. 169

[6] García Hernández J., Jordan F., Dubois J., Bolllat J.-L., Schleiss A. (2007) - Routing System II : Modélisation d'écoulements dans des systèmes hydrauliques. Communication du Laboratoire de Constructions Hydrauliques, Ed. A. Schleiss, EPFL, Lausanne. 32
[7] JORDAN F. (2007) - Modèle de prévision et de gestion des crues - optimisation des opérations des aménagements hydroélectriques à accumulation pour la réduction des débits de crue. PhD Thesis N 3711, Ecole Polytechnique Fédérale de Lausanne.

[8] Jordan F., Boillat J.-L., Garcia Hernandez J., Dubois J., Schleiss A. (2007) - Aide à la décision en situation de crue : le cas du Rhône en Valais. Forum für Wissen 2007. 79-83

[9] CHEN S.-T., Yu P.-S. (2007) - Real-time probabilistic forecasting of flood stages. Journal of Hydrology. 340 63-77

[10] Buizza R., Houtekamer P.L., Toth Z., Pellerin G., Wei M., ZHU Y. (2005) - A comparison of the ECMWF, MSC and NCEP global ensemble prediction systems. Monthly Weather Review. 133 1076-1097

[11] Demeritt D., Cloke H., Pappenberger F., Thielen J., BARTHOLMES J., RAMOS M.H. (2007) - Ensemble predictions and perceptions of risk, uncertainty and error in flood forecasting. Environmental Hazards. 7 115-127

[12] Marsigli C., Boccanera F., Montani A., Paccangnella T (2005) - The COSMO-LEPS ensemble system : validation of the methodology and verification. Nonlinear Processes in Geophysics. 12 527-536

[13] Marsigli C., Montani A., Paccangnella T. (2007) Ensemble activities at ARPA-SIM : the COSMO-LEPS and COSMO-SREPS systems. Proceedings, $29^{\text {th }}$ International Conference on Alpine Meteorology 4. - 8. June 2007, Chambéry.

[14] Obled C., Bontron G., Garcon R. (2002) - Quantitative precipitation forecasts : a statistical adaptation of model outputs through an analogues sorting approach. Atmospheric Research. 63(3-4) 303-324

[15] De Roo A., Gouneleeuw B., Thielen J., Bartholmes J., Bongioannini-Cerlini P., Todini E., Bates P., Horritt M., Hunter N., Beven K., Pappenberger F., Heise E., Rivin G., Hils M., Hollingsworth A., Holst B., Kwadijk J., Reggiani P., Van Dijk M., Sattler K., Sprokkereef E. (2003) 
- Development of a European flood forecasting system. International Journal of River Basin Management. 1 49-59

[16] Roulin E. (2007) — Skill and relative economic value of medium-range hydrological ensemble predictions. Hydrology and Earth System Sciences. 11 725-737

[17] Olsson J., Lindström G. (2008) — Evaluation and calibration of operational hydrological ensemble forecasts in Sweden. Journal of Hydrology. 350 14-24

[18] Faber M.H., Stewart M.G. (2003) - Risk assessment for civil engineering facilities : critical overview and discussion. Reliablility Engineering and System Safety. 80 173-184
[19] Arduino G., Reggiani P., Todini E. (2005) - Recent advances in flood forecasting and flood risk assessment. Hydrology and Earth System Sciences. 9(4) 280-284

[20] Cec, Commission Of The European Communities (2006) Proposal for a Directive of the European Parliament and of the European Council on the assessment and management of floods. Brussels 18.01.2006 SEC 66.

[21] Akter T., Simonovic S.P. (2005) - Aggregation of fuzzy views of a large number of stakeholders for multi-objective flood management decision-making. Journal of Environmental Management. 77 133-143 